\title{
Understanding the luminescent nature of organic radicals for efficient doublet emitters and pure-red light-emitting diodes
}

Alim Abdurahman ${ }^{1} \uparrow$, Timothy J. H. Hele ${ }^{2} \%$, Qinying Gu², Jiangbin Zhang ${ }^{2}$, Qiming Peng ${ }^{1,3}$, Ming Zhang $^{l}$, Richard H. Friend ${ }^{2 *}$, Feng Li ${ }^{1,2}$, Emrys W. Evans ${ }^{2 *}$

${ }^{1}$ State Key Laboratory of Supramolecular Structure and Materials, College of Chemistry, Jilin University, Qianjin Avenue 2699, Changchun, 130012, P. R. China

${ }^{2}$ Cavendish Laboratory, University of Cambridge, JJ Thomson Avenue, Cambridge, CB3 OHE, United Kingdom

${ }^{3}$ Key Laboratory of Flexible Electronics (KLOFE) \& Institute of Advanced Materials (IAM), Jiangsu National Synergetic Innovation Center for Advanced Materials (SICAM), Nanjing Tech University (NanjingTech), 30 South Puzhu Road, Nanjing 211816, P. R. China.

$\uparrow$ These authors contributed equally to this work.

* Authors to whom correspondence should be addressed;

rhf10@cam.ac.uk(RHF); lifeng01@jlu.edu.cn (FL); ee301@cam.ac.uk(EWE) 
The doublet-spin nature of radical emitters is advantageous for applications in organic light-emitting diodes (OLEDs), as it avoids the formation of triplet excitons that limit the electroluminescence efficiency of non-radical emitters. However, radicals generally show low optical absorption and photoluminescence yields. Here we explain the poor optical properties of radicals based on alternant hydrocarbons, and establish design rules to increase absorption and luminescence yields for donor-acceptor-type radicals. We show that non-alternant systems are necessary to lift the degeneracy of the lowest energy orbital excitations; moreover, intensity borrowing from an intense high-lying transition by the low energy chargetransfer excitation enhances the oscillator strength of the emitter. We apply these rules to design tris[2,4,6trichlorophenyl] methyl (TTM)-pyridoindolyl derivatives with high photoluminescence quantum yield (>90\%). OLEDs based on these molecules showed pure-red emission with over $12 \%$ external quantum efficiency. These insights may be beneficial for the rational design and discovery of highly luminescent doublet emitters. 
In general, closed-shell materials are employed in organic light-emitting diodes (OLEDs), where the electrons are paired and the molecular orbitals are filled up to the highest occupied molecular orbital (HOMO) by the Aufbau Principle. Following electrical excitation, electrons are injected into the lowest unoccupied molecular orbital (LUMO) and abstracted from the HOMO to form 25\% singlet (spin-0) and $75 \%$ triplet (spin1) states. Given a singlet ground state, the well-known spin statistics problem dictates that the maximum electroluminescence (EL) efficiency is limited to $25 \%$ as singlet and triplet states are intrinsically bright and dark, respectively. In order to overcome this, triplet states have been brightened directly, by inclusion of transition metals for promoting phosphorescence via heavy metal-enhanced spin-orbit coupling ${ }^{1-3}$; and indirectly, by Etype (or thermally activated delayed fluorescence, $\mathrm{TADF}^{4}$ ) and P-type delayed fluorescence (or triplet-triplet annihilation $\left.{ }^{5,6}\right)$.

With organic radicals, a different approach to triplet management in OLEDs has emerged. Most applications of stable radicals have focused on their magnetic and electron transfer properties for molecular magnets ${ }^{7,8}$, batteries $^{9}$, organic field-effect transistors (OFETs) ${ }^{10,11}$, improving conductivity of electrical devices ${ }^{12}$, charge mediators in solar cells ${ }^{13}$ and accelerating chemical reactions ${ }^{14}$. Reports of radical-based photo- ${ }^{15-19}$ and electroluminescence $\mathrm{e}^{20-22}$ make them exciting prospects for the basis of next-generation optoelectronics. The singly occupied molecular orbital (SOMO) dictates that both the ground and first excited states must have doublet-spin character and that emission is totally spin allowed (see fluorescence, $\mathrm{D}_{1} \rightarrow \mathrm{D}_{0}$ ) $^{23-25}$. Further, the SOMO can eliminate triplets from the functional device physics in radical-based OLEDs, and our recent work showed that such OLEDs can be operated entirely within the doublet exciton manifold ${ }^{21}$. In some cases, non-Aufbau HOMOSOMO inversion of the frontier orbitals can be exploited for improved radical stability ${ }^{26}$.

To date, success for radical-based OLEDs has been achieved by covalent attachment of electron donor groups to tris(2,4,6-trichlorophenyl)methyl (TTM) radicals and perchloro-substituted variants ${ }^{21,26}$. As radicals are associated with quenched luminescence by electron transfer and spin-exchange interactions, that TTM-donor derivatives can have such efficient light emission (photoluminescence quantum efficiency, PLQE $=85.6 \%$ ) and nanosecond luminescence lifetimes (17.2 ns) is surprising, but highly attractive for OLED applications ${ }^{21}$. Current discussions on the nature of emission in these radicals are mainly derived from unrestricted Kohn Sham density functional theory (UKS-DFT), and highlight the HOMO-donor to SOMO-radical-acceptor electronic transition for the fluorescent doublet exciton $\left(D_{1}\right)^{27,28}$. These studies suggest that greater HOMO-SOMO overlap should 
promote higher oscillator strength for more rapid radical emission. However, they do not address the fundamental reasons as to why TTM and similar radicals are dark, yet the TTM-donor derivatives can be bright? We shed light on this question and have formulated general design rules for obtaining luminescent radicals, demonstrating that significant orbital overlap is not a sufficient criterion. Our model allows us to make directed chemical alterations to TTM-carbazole derivatives to control the colour of the radicals, yielding pure red emitters that are optimised for the 'red' in red-green-blue displays.

\section{Electronic structure considerations for radical-based luminescence}

The TTM radical has a weak absorption in the visible $\left(\varepsilon=850 \mathrm{M}^{-1} \mathrm{~cm}^{-1} \text { at } 544 \mathrm{~nm}\right)^{15}$, and a strong absorption in the UV ( $\varepsilon=38150 \mathrm{M}^{-1} \mathrm{~cm}^{-1}$ at $\left.374 \mathrm{~nm}\right)$. It is only weakly luminescent: with photoluminescence at $566 \mathrm{~nm}$ in $\mathrm{CHCl}_{3}$ (Fig. 1b), and measured photoluminescence quantum efficiency (PLQE) of $2.6 \%$ and radiative rate of $10^{6}$ $\mathrm{s}^{-1}$. These properties reflect the relatively weak extinction coefficient for the $\mathrm{D}_{0} \rightarrow \mathrm{D}_{1}$ absorption in TTM, signifying a low oscillator strength for the lowest energy electronic transition which also translates to slow radiative emission in all solvents (Table S4 in SI 5).

In fact, the TTM radical's poor emissive properties are general features for alternant hydrocarbon systems where conjugated atoms can be divided into two sets and that no two atoms of the same set are directly linked. In such systems, the HOMO-SOMO and SOMO-LUMO energy gaps were proven to be identical ${ }^{29,30}$. The HOMO-SOMO $\left|\Psi_{\mathrm{hT}}^{\mathrm{sT}}\right\rangle$ and SOMO-LUMO $\left|\Psi_{\mathrm{ST}}^{\mathrm{IT}}\right\rangle$ TTM-based excitations are degenerate and also have identical dipole moments (Fig. 1c,1d). These two degenerate excitations are mixed by the electronic Hamiltonian to form an out-of-phase excitation at lower energy $\left|\mathrm{D}_{1}\right\rangle$ with minimal oscillator strength (seen at $544 \mathrm{~nm}$ for TTM in Fig. 1b), and an in-phase combination at higher energy $\left|D_{2}\right\rangle$ with high oscillator strength (seen as an intense absorption for TTM at $374 \mathrm{~nm}$ ). Although the previous researchers did not consider OLED applications, their proof suggests that it would be difficult to make an efficient radical emitter based on an alternant hydrocarbon ${ }^{29,30}$, since emission from $\mathrm{D}_{1}$ would be weak and associated with a slow radiative rate that would be outcompeted by non-radiative decay. The proof is based on an electronic structure model similar to Pariser-Parr-Pople theory ${ }^{31-34}$ for closed-shell molecules, which has the simplicity of Hückel theory, but unlike Hückel theory also includes two-electron (Coulomb and exchange) interactions for accurate description of excited states. Note that the 
HOMO-SOMO and SOMO-LUMO energy gap degeneracy is not necessarily found in density functional theory calculations which are usually employed to study the radicals' electronic structure ${ }^{21,23,27}$.

\section{Figure 1}


Fig. 1. Nature of light emission from radicals based on alternant and non-alternant hydrocarbons.

a) Chemical structures of the TTM (alternant) and TTM-1Cz (non-alternant). b) Optical absorption (solid lines) and fluorescence (dotted lines) spectra of the TTM and TTM-1Cz radicals in $\mathrm{CHCl}_{3}$. c) Schematic molecular orbital diagram of the TTM and carbazole moieties in TTM-1Cz, with electron occupancy shown by half-headed arrows. d) Transition dipole moments (TDM) for HOMO-SOMO $\left|\Psi_{\mathrm{hT}}^{\mathrm{sT}}\right\rangle$ and SOMO-LUMO $\left|\Psi_{\mathrm{ST}}^{\mathrm{IT}}\right\rangle$ transitions in TTM (blue arrows), showing out-of-phase and in-phase combinations for the $\left|\mathrm{D}_{1 \text { (TTM) }}\right\rangle$ and $\left|\mathrm{D}_{2 \text { (TTM) }}\right\rangle$ excited states. Note that the indicated $\left|\Psi_{\mathrm{hT}}^{\mathrm{ST}}\right\rangle$ and $\left|\Psi_{\mathrm{sT}}^{\mathrm{IT}}\right\rangle$ excitations are one pair from a degenerate set; the other excitation pair has horizontal dipole moments. e) The weak CT transition dipole moment of TTM-1Cz is enhanced by intensity borrowing from the TTM moiety's bright $\left|\mathrm{D}_{2(\mathrm{TTM})}\right\rangle$ state.

It is not a coincidence that the main route to stable and luminescent radicals has been by covalent attachment of electron-rich, non-alternant hydrocarbon groups to TTM and perchlorotriphenylmethyl (PTM), forming nonalternant-donor-acceptor radicals ${ }^{15-18,21,26,27,35,36}$ (SI 6). Radicals should be synthesised with broken alternacy symmetry which, to the best of our knowledge, was not considered possible ${ }^{29,30}$. In TTM-1Cz ([4-( $N$-carbazolyl)2,6-dichlorophenyl]bis(2,4,6-trichlorophenyl)methyl ${ }^{15}$, Fig. 1c), considered a breakthrough molecule for radical luminescence, the $\mathrm{D}_{1}$ excited state is a carbazole HOMO $\left(\mathrm{h}_{\mathrm{Cz}}\right)$ to TTM SOMO $\left(\mathrm{s}_{\mathrm{T}}\right)$ electronic transition with charge transfer (CT) character. The colour of light emission is then related to the $\mathrm{h}_{\mathrm{Cz}}-\mathrm{s}_{\mathrm{T}}$ energy gap (Fig. 1c), and is $687 \mathrm{~nm}$ in $\mathrm{CHCl}_{3}$ (Fig. 1b) ${ }^{15}$. Breaking the alternacy symmetry leads to a substantial increase in the extinction 
coefficient of the lowest excited state of TTM-1Cz $\left(3780 \mathrm{M}^{-1} \mathrm{~cm}^{-1}\right.$, Fig. 1b) compared to TTM $\left(850 \mathrm{M}^{-1} \mathrm{~cm}^{-1}\right)$. A higher $\mathrm{D}_{1}$ transition dipole moment leads to roughly twenty- and four-fold increases in PLQE and radiative rates, respectively, for TTM-1Cz versus TTM in cyclohexane: $2 \%$ to $53 \%$ and $3.5 \times 10^{6} \mathrm{~s}^{-1}$ to $1.3 \times 10^{7} \mathrm{~s}^{-1}$.

Whilst the weak $\mathrm{D}_{1}$ transition for alternant radical systems is circumvented in TTM-1Cz and other donoracceptor-type radicals, since the $\mathrm{D}_{1}$ transition has significant charge-transfer character one might expect the oscillator strength to be inherently weak. To zeroth order, CT transitions are dark since the relevant orbitals do not overlap in space, and so $\left\langle\mathrm{D}_{0(\mathrm{TTM}-1 \mathrm{Cz})}^{(0)}|\hat{\mu}| \mathrm{D}_{1(\mathrm{TTM}-1 \mathrm{Cz})}^{(0)}\right\rangle=0 .{ }^{37}$ However, to first order, the CT state can 'borrow intensity' from TTM moiety's bright $\left|\mathrm{D}_{2(\mathrm{TTM})}^{(0)}\right\rangle$ state. This phenomenon is supported experimentally: careful examination of Fig. $1 \mathrm{~b}$ shows that, whilst the $\mathrm{D}_{1}$ extinction coefficient of TTM-1Cz is greater than that of TTM, there is a concomitant decrease in the intensity of the strong $\mathrm{D}_{2}$ transition at $374 \mathrm{~nm}$ from $\varepsilon=38150 \mathrm{M}^{-}$ ${ }^{1} \mathrm{~cm}^{-1}$ in TTM to $32670 \mathrm{M}^{-1} \mathrm{~cm}^{-1}$ in TTM-1Cz. More formally, using intensity borrowing perturbation theory ${ }^{38}$ :

$$
\left|\mathrm{D}_{1(\mathrm{TTM}-1 \mathrm{Cz})}^{(1)}\right\rangle=\left|\mathrm{D}_{1(\mathrm{TTM}-1 \mathrm{Cz})}^{(0)}\right\rangle+X\left|\mathrm{D}_{2(\mathrm{TTM})}^{(0)}\right\rangle
$$

where

$$
X=\frac{\left\langle\mathrm{D}_{2(\mathrm{TTM})}^{(0)}|\hat{V}| \mathrm{D}_{1(\mathrm{TTM}-1 \mathrm{Cz})}^{(0)}\right\rangle}{E\left(\mathrm{D}_{1(\mathrm{TTM}-1 \mathrm{Cz})}^{(0)}\right)-E\left(\mathrm{D}_{2(\mathrm{TTM})}^{(0)}\right)}
$$

and $\widehat{V}$ corresponds to the electronic coupling perturbation Hamiltonian, and $E(y)$ the energy for the $y=$ $\mathrm{D}_{2(\mathrm{TTM})}^{(0)}$ and $\mathrm{D}_{1(\mathrm{TTM}-1 \mathrm{Cz})}^{(0)}$ states. Given the local excited and CT character for $\left|\mathrm{D}_{2(\mathrm{TTM})}^{(0)}\right\rangle$ and $\left|\mathrm{D}_{1(\mathrm{TTM}-1 \mathrm{Cz})}^{(0)}\right\rangle$ excitations, respectively, non-zero matrix elements for the mixing term are expected for orbital coefficients associated with atoms bonding the TTM and carbazole moieties. Approximating the electronic structure of the radical using Longuet-Higgins' model ${ }^{37,38}$,

$$
\left\langle\mathrm{D}_{2(\mathrm{TTM})}^{(0)}|\widehat{V}| \mathrm{D}_{1(\mathrm{TTM}-1 \mathrm{Cz})}^{(0)}\right\rangle \approx-\frac{1}{\sqrt{2}} \beta C_{T *, i} C_{C Z *, j} \cos \theta,
$$

where $\beta$ is the Hückel hopping term; $\theta$ is the dihedral angle between radical and adjacent non-radical group; $C_{T * i}$ is the TTM HOMO coefficient of orbital $i$ on joining atom $T^{*}$ (*carbon atom on TTM moiety, Fig. 1e), and likewise $C_{C Z *, j}$ for the carbazole HOMO coefficient of orbital $j$ on joining atom $C z^{*}$ (*nitrogen atom on carbazole moiety, Fig. 1e). Intuitively, the cosine term suggests that intensity borrowing - and therefore hybridisation of the excited states - is enhanced upon planarisation of the TTM-radical and carbazole-donor $\pi$ systems. This consolidates the orbital delocalisation picture for radical-SOMO and donor-HOMO as presented 
by Rovira and Cornil et al. ${ }^{27}$. Analogously, intensity borrowing of oscillator strength can occur where the radical acts as an electron donor in $\mathrm{D}_{1}$, and now the relevant orbitals $i$ and $j$ would be TTM-LUMO $\left(1_{\mathrm{T}}\right)$ and acceptor group LUMO.

In summary, we can formulate two general design rules for obtaining luminescent donor-acceptor-type radicals: i) the radical emitter should be a non-alternant hydrocarbon; ii) for intensity borrowing of oscillator strength in electron-donor/radical-electron-acceptor emitters, there must be significant orbital amplitudes on the atoms joining the radical HOMO and electron donor HOMO; alternatively, electron-acceptor/radical-electrondonor species require significant orbital amplitudes on the atoms joining the radical LUMO and electron acceptor LUMO.

While design rule ii) can be loosely interpreted as orbital interaction of the donor/acceptor $\pi$-systems leading to mixing and light emission, orbital interaction alone is not sufficient for efficient luminescence if the emitter is still an alternant hydrocarbon. In other words, criterion i) must be satisfied as well as criterion ii). For example, if an alternant hydrocarbon such as tetracene is attached to TTM (SI 7, Fig. S4a), the resultant TTM-Tetracene radical will also be alternant. The result is that the lowest energy excitation in TTM-Tetracene, like TTM, is comprised of an out-of-phase combination of transition dipoles, and leads to a weak oscillator strength for light emission (SI 7).

Our general design principles can be used in conjunction with molecular discovery methods to uncover new radical emitters, supporting the assessment of suitable candidates for more detailed quantum chemical calculations, and avoiding wasted efforts in synthesising inherently non-emissive radicals.

\section{Design of the pure-red radical emitter TTM-xPyID}

Following the theoretical framework above, we directed efforts to design a series of radicals with pure-red emission around $630 \mathrm{~nm}$ and Commission Internationale de L'Eclairage (CIE) chromaticity coordinates of $[0.67,0.33]$. From the simple picture in Fig. 1c, we previously noted that the emission energy of the TTM-1Cz radical is related to the $\mathrm{h}_{\mathrm{Cz}}$ - $\mathrm{S}_{\mathrm{T}}$ energy gap. Consequently, the emission can be blue-shifted by either raising the energy of the TTM SOMO or lowering the energy of the carbazole HOMO, preferably with the smallest possible chemical alteration. Taking the latter approach, we considered singular aza-substitution of the TTM-1Cz 
carbazole group, for which there are four symmetry-unique sites, leading to four TTM-pyridoindolyl derivatives: TTM- $\alpha$ PyID, TTM- $\beta$ PyID, TTM- $\gamma$ PyID and TTM- $\delta$ PyID (Fig. 2a).
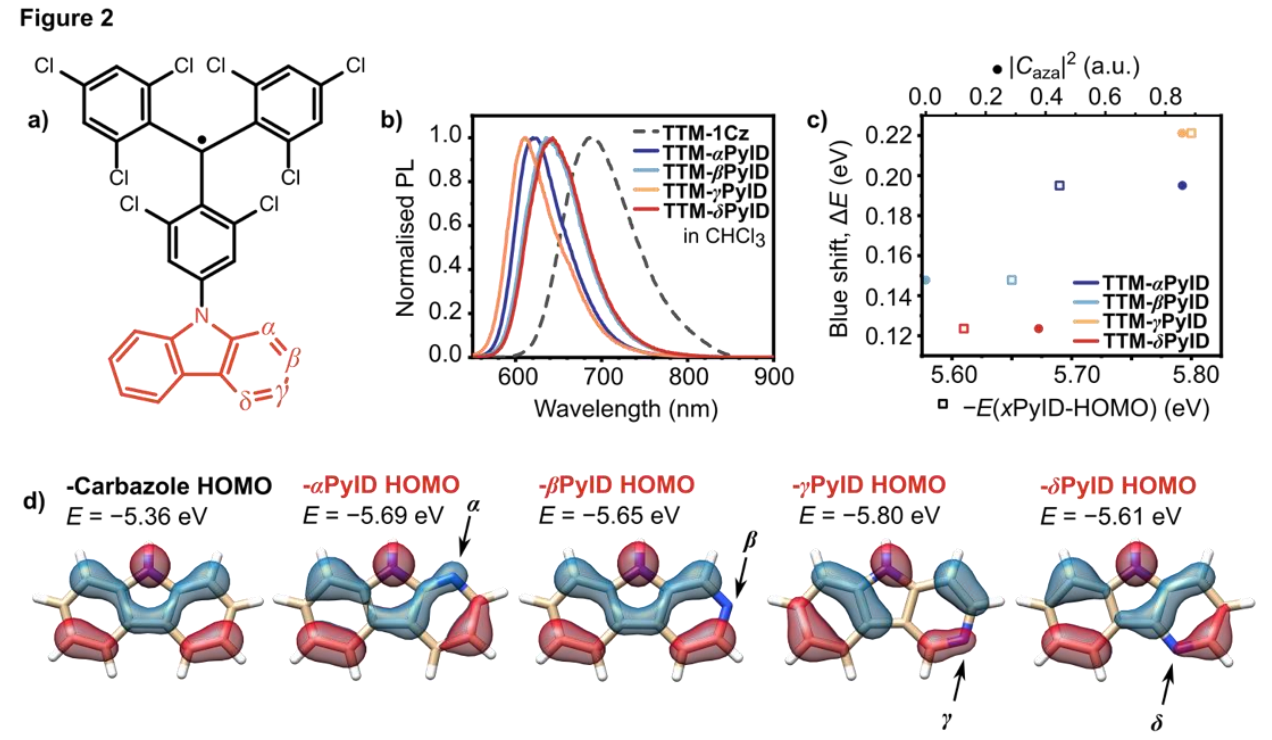

Fig. 2. Red emission design for TTM-based radicals. a) Chemical structures of the TTM- $x$ PyID with carbazole aza-substitution at site $x=\alpha, \beta, \gamma, \delta$. b) The fluorescence spectra of the TTM-1Cz and azasubstituted radicals (TTM- $x$ PyID) in $\mathrm{CHCl}_{3}$. c) Blue shift of the emission versus carbazole HOMO orbital coefficient at aza-position (filled circles) and $x$ PyID HOMO orbital energies (open squares). d) DFT-calculated $\mathrm{HOMO}$ energies and orbital coefficients for $-\mathrm{Cz},-\alpha \mathrm{PyID},-\beta \mathrm{PyID},-\gamma \mathrm{PyID}$ and $-\delta$ PyID moieties (isovalue $=$ 0.035 ; red and blue denote different phases for the orbital wave function).

The blue-shifting can be understood as the $\mathrm{N}$ atom's higher electronegativity versus $\mathrm{C}$ leads to lowering the energy of the carbazole-HOMO upon aza-substitution, which increases the critical $\mathrm{h}_{\mathrm{Cz}}$-s $\mathrm{s}$ energy gap for photoluminescence. By first order perturbation theory in a Hückel framework, the blue shift of the TTM-xPyID photoluminescence versus TTM-1Cz follows:

$$
\Delta E=\Delta \alpha \sum_{v}\left|C_{v}\right|^{2}=\Delta \alpha\left|C_{\mathrm{aza}}\right|^{2}
$$

where $\Delta \alpha$ is the change in the on-site energy (Hückel $\alpha$ term) following exchange of sp ${ }^{2}$-hybridised $\mathrm{C}$ and $\mathrm{H}$ to $\mathrm{N}$, and $C_{v}$ is the HOMO coefficient of the $v$-substituted carbon atom. For the $x$ PyID series, we have one site of aza-substitution with carbazole orbital coefficient denoted $C_{\text {aza }}$. From Eq. 2, the largest blue-shift is therefore expected for aza-substitution at the sites with largest HOMO coefficients on carbazole, which can be evaluated by density functional theory (DFT) with KS method and B3LYP, 6-31G** (Fig. 2d). Here, the predicted blueshifting is TTM- $\alpha$ PyID $\approx$ TTM- $\gamma$ PyID $>$ TTM- $\delta$ PyID $>$ TTM- $\beta$ PyID. 
We synthesised and characterised the TTM-xPyID radicals, details of which can be found in SI 1. Electron paramagnetic measurements verified the doublet-spin nature of these materials (SI 8). We find that singular azasubstitution has a profound effect and blue-shifts the peak photoluminescence of $687 \mathrm{~nm}$ found for TTM-1Cz in $\mathrm{CHCl}_{3}$ (Fig. 2b), verifying our theoretical prediction. The experimentally-observed order of blue-shifting follows: TTM- $\gamma$ PyID $(612 \mathrm{~nm})>$ TTM- $\alpha$ PyID $(620 \mathrm{~nm})>$ TTM- $\beta$ PyID $(635 \mathrm{~nm})>$ TTM- $\delta$ PyID $(643 \mathrm{~nm})$, matching exactly the predictions from DFT calculations of substituted carbazole HOMO energies (Fig. 2d), and approximately those from a simple Hückel picture.

In order to evaluate our predictions more quantitatively, in Fig. $2 \mathrm{c}$ we plot the experimentally-observed blue shift $\Delta E$ against the square of the HOMO coefficient from Eq. 2 (filled circles), and against the DFT-calculated pyridoindolyl HOMOs from Fig. 2d (open squares). The crude Hückel predictions are improved by DFT calculations of the - $x$ PyID HOMO energies, as the former approach does not account for orbital structure changes following substitution. Full electronic structure calculations for the TTM-xPyID radicals are given in SI 9, and reproduce the same general trends found for the -xPyID fragments in Fig. 2c,d.

In favour of light emission, aza-substitution also leads to much enhanced photoluminescence quantum yield in $\mathrm{CHCl}_{3}$ for TTM- $\alpha$ PyID (91\%), TTM- $\beta$ PyID (89\%), TTM- $\gamma$ PyID (32\%) and TTM- $\delta$ PyID (99\%) vs TTM- $1 \mathrm{Cz}$ (5\%). This trend is observed for the TTM-xPyID series in all solvents (SI 4) when compared with TTM-1Cz (apart from TTM- $\gamma$ PyID instability). Favourably, aza-substitution yields decreased non-radiative rates compared to TTM-1Cz due to the Energy Gap Law (SI 5), as reduced non-adiabatic coupling occurs with increasing $\mathrm{s}_{\mathrm{T}}-\mathrm{h}_{\mathrm{Cz}}$ energy gap.

It is noteworthy that the PLQEs of TTM-xPyID radicals are mostly independent of solvent polarity (SI 4, Table S1). From Eq. 1, we can see that $\mathrm{D}_{1}$ of TTM-xPyID radicals is a hybridised local and charge transfer (HLCT) excited state which originates from intensity borrowing ${ }^{39}$. This suggests that the local excited nature of $\mathrm{D}_{1}$ also plays an important role besides the CT character in donor-acceptor radical emitters. More detailed discussion of solvent polarity effects can be found in SI 5. Additionally, our general model for the donor-acceptortype radical's electronic structure is supported by protonation studies of TTM- $\beta$ PyID which reversibly alter the nature of $\mathrm{D}_{1}$ emission: switching the radical from electron acceptor to donor, and the emitter from SOMOHOMO-type to LUMO-SOMO-type (SI 10). 


\section{Performance of TTM-xPyID radical-based light-emitting diodes}

High PLQE, good photo- and redox- stabilities (SI 11,12), and nanosecond light emission around 612-643 $\mathrm{nm}$, make these molecules ideal candidates for pure-red emitters of commercial interest in display technologies. Devices were fabricated using the traditional OLED approach of high vacuum deposition $\left(<10^{-7}\right.$ Torr $)$ whereby the radical-containing emissive layers were evaporated at $<150^{\circ} \mathrm{c}$ for the radicals, lower than the $95 \%$ temperature stability point (SI 13). From a series of chemical composition tests to determine the purity of deposited radical films, we confirmed the suitability of the vacuum deposition method for these materials (SI 14). The overall stability of the radicals may be attributed to some protection from photocyclisation reactions, deterred by the CT nature of the excited state $\mathrm{A0}^{40}$. The OLED architecture used for the TTM-xPyID series was (Fig. 3a): ITO / $\mathrm{MoO}_{3}(3 \mathrm{~nm}) /$ TAPC (40 nm) / 4\% TTM-xPyID in TPBi (30 nm) / B3PYMPM (60 nm) / LiF (0.8 nm) / $\mathrm{Al}(100 \mathrm{~nm})$.
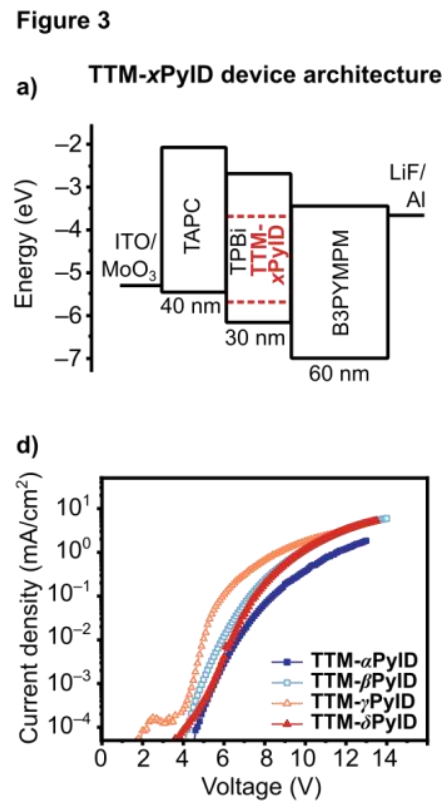

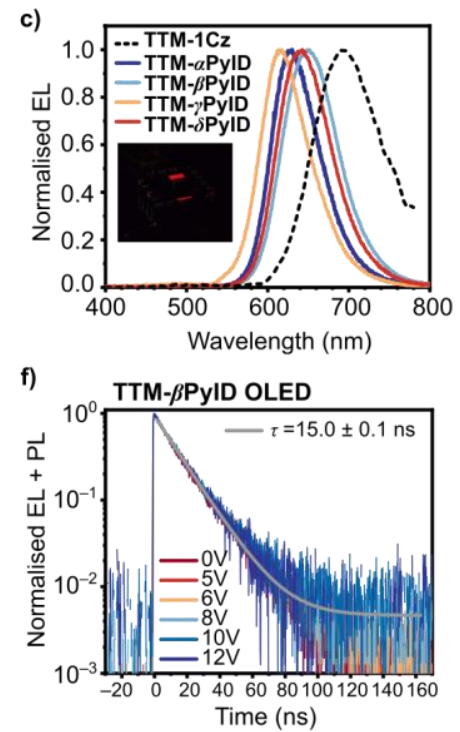

Fig. 3. Optoelectronic performance of the TTM-XPyID radical-based OLEDs. a) Device architecture for OLEDs based on the TTM- $x$ PyID series. b) EQE-current density, c) EL spectra (inset photograph of TTM- $\beta$ PyID device), d) current density-voltage and e) luminance-voltage curves for TTM-xPyID OLEDs. f) Time-resolved PL plots for TTM- $\beta$ PyID OLEDs at 0V, 5V, 6V, 8V, $10 \mathrm{~V}$ and $12 \mathrm{~V}(200 \mathrm{kHz}, 2 \mu$ s electrical pulse). A $407 \mathrm{~nm}$ laser pulse (200 kHz, 50 ps pulse width) was synchronised to arrive in the middle of each electrical pulse. The PL plots have been normalised following subtraction of EL contributions.

The performance of TTM- $x$ PyID-based devices are given in Fig. 3b-e. We were able to translate favourable photoluminescence properties to highly efficient OLEDs with maximum EQE value over $12 \%$ : TTM- $\alpha$ PyID 
$(\max \mathrm{EQE}=9.6 \%$, peak $=629 \mathrm{~nm}) ; \mathrm{TTM}-\beta \operatorname{PyID}(12.2 \%, 649 \mathrm{~nm}) ; \mathrm{TTM}-\gamma \mathrm{PyID}(2.9 \%, 616 \mathrm{~nm}) ; \mathrm{TTM}-\delta$ PyID $(9.5 \%, 641 \mathrm{~nm})$. The EQE values $(\geq 10 \%)$ signify the state of the art for radical-based OLEDs with doublet emission in the visible range, and are higher than the conventional singlet-triplet spin statistics limit $(\mathrm{EQE}=6$ 8\%; from $25 \%$ singlet yield, $100 \%$ PLQE and 25-33\% light out-coupling). Importantly, the EL spectra were found to match up well with pure-red CIE coordinates [0.67,0.33]: TTM- $\alpha$ PyID [0.66,0.34]; TTM- $\beta$ PyID [0.67,0.32]; TTM- $\gamma$ PyID [0.61,0.37]; TTM- $\delta$ PyID [0.67,0.33]. See Fig. 3b and 3c for EQE-current density profiles and EL spectra, which show a step change in OLED performance and significant blue-shift with respect to TTM-1Cz ${ }^{19}$. Note that the EL spectra do not exhibit TPBi-host emission at any voltage (SI 15), supporting a charge trapping rather than host-to-radical dopant energy transfer mechanism for emission. The voltage-current density-luminance curves are plotted in Fig. 3d and 3e, and further information on the devices can be found in SI 15. Being organic materials, the TTM-xPyID radical emitters also offer the opportunity for low-cost and lowenergy, ink-type fabrication of devices. We fabricated solution-processed OLEDs based on TTM- $\beta$ PyID with reasonably high efficiency of up to $4.0 \%$ (SI 16).

The current radical-based OLEDs do not sustain their maximum EQE values well beyond the turn on. The critical current density $J_{0}$ which corresponds to device performance at half the maximum EQE is $0.3-0.4 \mathrm{~mA} / \mathrm{cm}^{2}$ for the TTM- $\alpha$ PyID, TTM- $\beta$ PyID, TTM- $\gamma$ PyID and TTM- $\delta$ PyID devices (Fig. $3 b$ ). As the radicals have nanosecond lifetimes associated with radiative emission, reduced exciton quenching in efficiency roll-off should be possible in improved devices when compared with the microsecond emission and excited state lifetimes found for phosphorescent, triplet fusion and TADF devices ${ }^{43}$.

PL transients were recorded for electrically driven TTM- $\beta$ PyID OLEDs to probe the exciton quenching mechanisms in devices with relevant charge and exciton densities for operation ${ }^{44}$. From these studies, we can identify the relative contributions to roll-off by exciton-exciton quenching $\left(D_{1}+D_{1} \rightarrow D_{x}+D_{0}\right)$, exciton-charge annihilation $\left(D_{1}+e^{-/} / h^{+} \rightarrow D_{0}+e^{*} / h^{*}\right)$, charge imbalance and electric field effects ${ }^{43}$. In these studies, TTM$\beta$ PyID devices (Fig. 3a) were subjected to $2 \mu$ s electrical pulses at $200 \mathrm{kHz}$, with varied voltage from 5 to $12 \mathrm{~V}$ (corresponding to current densities of $\mu \mathrm{A} / \mathrm{cm}^{2}-\mathrm{mA} / \mathrm{cm}^{2}$, Fig. 3d). A $407 \mathrm{~nm}$ laser pulse was synchronised to arrive in the middle of each electrical pulse. As would be expected, increasing voltage leads to an increased EL signal over which the PL transients are superimposed (SI 17, Fig. S21). Following subtraction of the EL signals, we found that the normalised transients obtained at different applied voltages could be perfectly overlaid (Fig. 
3f). This provides evidence that exciton-exciton quenching should not be the cause of the roll-off, and also rules out exciton-charge annihilation and electric field effects (on the nanosecond emission timescale); all of these mechanisms would have led to decreasing PL transient lifetimes with increasing voltage/current density. Significant exciton-exciton quenching was additionally ruled out from the PL intensity power dependence for TTM- $\beta$ PyID films (SI 18).

We therefore attribute the roll-off in the radical devices to charge imbalance. As we noted in our previous work $^{21}$, electrical excitation of radicals for light emission requires electron and hole injection into the SOMO and HOMO respectively. One possible loss mechanism would be the scenario of both electrons and holes being injected onto the SOMO, which would lead to no electroluminescence. Overcoming such losses, in combination with the avoided spin statistics limit and more rapid nanosecond emission lifetimes than microsecond phosphorescent and TADF materials, will lead to further opportunities to advance the state of the art for radicalbased OLEDs and organic electronics.

In conclusion, we have shown how unexpectedly luminescent organic radicals can be constructed from the well-known, but dark, TTM radical by breaking the alternant hydrocarbon nature of its $\pi$-system and lifting the HOMO-SOMO and SOMO-LUMO energy gap degeneracy. Aza-substitution of the carbazole moiety in TTM$1 \mathrm{Cz}$ boosted the radicals' photoluminescence properties to over 90\% PLQE and blue-shifted its emission by up to $220 \mathrm{meV}$ in $\mathrm{CHCl}_{3}$. From the TTM-xPyID series, we fabricated devices with up to $12 \%$ electroluminescence EQE and excellent matching of the ideal red CIE [0.67,0.33] coordinate. These materials are promising candidates for radical-based red light in red-green-blue displays. More generally, we can summarise two design considerations for obtaining luminescent donor-acceptor-type radicals: i) the radical emitter must be a nonalternant hydrocarbon; ii) intensity borrowing from higher energy transitions can increase the oscillator strength of charge-transfer-type emission. The open-shell radicals' charge-transfer-type excited states appear similar to that of closed-shell TADF materials which also have donor-acceptor motifs. However, there is a trade-off between high oscillator strength and low spin-exchange interaction for efficient reverse intersystem crossing from dark triplet to bright singlet excitons in TADF materials, which require strong and weak overlap of the donor and acceptor wavefunctions, respectively. This does not apply to donor-acceptor-type radicals because the lowest excited state is bright, thus intersystem crossing from dark to bright states is not necessary. The search for doublet materials geared to blue and green light emission will benefit from the theoretical concepts presented here which 
provides guidelines for the discovery and utilisation of new radical motifs for next-generation display and lighting applications.

\section{Acknowledgements}

AA, QP, MZ and FL are grateful for financial support from the National Natural Science Foundation of China (grant no. 51925303, 91833304 and 61935017), the National Key R\&D Programme of China (grant no. 2016YFB0401001) and the programme 'JLUSTIRT' (grant no. 2019TD-33). TJHH thanks Jesus College, Cambridge for a Research Fellowship. JZ and QG thanks the China Scholarship Council for a PhD scholarship (no. 201503170255). RHF and EWE would like to thank the EPSRC for funding this work (EP/M005143/1). EWE also acknowledges support from the Leverhulme Trust and Newton Trust. FL is an academic visitor at the Cavendish Laboratory, Cambridge, and is supported by the Talents Cultivation Programme (Jilin University, China).

\section{Author contributions}

AA and MZ designed, synthesised and characterised the luminescent radicals. AA, QG, JZ, QP, FL and EWE optimised the devices. TJHH and EWE devised the theoretical treatment and performed the electronic structure calculations. RHF, FL and EWE conceived the project, supervised the work and wrote the manuscript with input from all authors.

\section{Additional information}

Supplementary information accompanies this paper at [to be completed in proofs]. Datasets associated with this work are available at 10.6084/m9.figshare.12229448.

\section{Competing financial interests}

The authors declare no competing interests.

\section{References}

1. Baldo, M. A. et al. Highly efficient phosphorescent emission from organic electroluminescent devices. Nature 395, 151 (1998).

2. Ma, Y., Zhang, H., Shen, J. \& Che, C. Electroluminescence from triplet metal-ligand charge-transfer excited state of transition metal complexes. Synth. Met. 94, 245-248 (1998). 
3. Adachi, C., Baldo, M. A., Thompson, M. E. \& Forrest, S. R. Nearly $100 \%$ internal phosphorescence efficiency in an organic light-emitting device. J. Appl. Phys. 90, 5048-5051 (2001).

4. Uoyama, H., Goushi, K., Shizu, K., Nomura, H. \& Adachi, C. Highly efficient organic light-emitting diodes from delayed fluorescence. Nature 492, 234 (2012).

5. Kido, J. \& Lizumi, Y. Fabrication of highly efficient organic electroluminescent devices. Appl. Phys. Lett. 73, 2721 (1998).

6. Di, D. et al. Efficient triplet exciton fusion in molecularly doped polymer light-emitting diodes. Adv. Mater. 29, 1605987 (2017).

7. Awaga, K. \& Maruyama, Y. Ferromagnetic and antiferromagnetic intermolecular interactions of organic radicals, $\alpha$ nitronyl nitroxides. II. J. Chem. Phys. 91, 2743-2747 (1989).

8. Banister, A. J. et al. Spontaneous magnetization in a sulfur-nitrogen radical at 36 K. Angew. Chem. Int. Ed. 35, 2533-2535 (1996).

9. Nakahara, K. et al. Rechargeable batteries with organic radical cathodes. Chem. Phys. Lett. 359, 351-354 (2002).

10. Wang, Y. et al. 1-Imino nitroxide pyrene for high performance organic field-effect transistors with low operating voltage.

J. Am. Chem. Soc. 128, 13058-13059 (2006).

11. Wei, P., Oh, J. H., Dong, G. \& Bao, Z. Use of a 1 H-benzoimidazole derivative as an n-type dopant and to enable air-stable solution-processed n-channel organic thin-film transistors. J. Am. Chem. Soc. 132, 8852-8853 (2010).

12. Bin, Z., Duan, L. \& Qiu, Y. Air stable organic salt as an n-type dopant for efficient and stable organic light-emitting diodes. ACS Appl. Mater. Interfaces 7, 6444-6450 (2015).

13. Zhang, Z., Chen, P., Murakami, T. N., Zakeeruddin, S. M. \& Grätzel, M. The 2, 2, 6, 6-tetramethyl-1-piperidinyloxy radical: An efficient, iodine-free redox mediator for dye-sensitized solar cells. Adv. Funct. Mater. 18, 341-346 (2008).

14. Jiao, Y. et al. A supramolecularly activated radical cation for accelerated catalytic oxidation. Angew. Chem. Int. Ed. 55, 8933-8937 (2016).

15. Gamero, V. et al. [4-(N-carbazolyl)-2, 6-dichlorophenyl] bis (2, 4, 6-trichlorophenyl) methyl radical an efficient red lightemitting paramagnetic molecule. Tetrahedron lett. 47, 2305-2309 (2006).

16. Velasco, D. et al. Red organic light-emitting radical adducts of carbazole and tris(2,4,6-trichlorotriphenyl)methyl radical that exhibit high thermal stability and electrochemical amphotericity. J. Org. Chem. 72, 7523-7532 (2007).

17. Heckmann, A., Lambert, C., Goebel, M. \& Wortmann, R. Synthesis and photophysics of a neutral organic mixed-valence compound. Angew. Chem. Int. Ed. 43, 5851-5856 (2004).

18. Heckmann, A. \& Lambert, C. Neutral organic mixed-valence compounds: synthesis and all-optical evaluation of electron-transfer parameters. J. Am. Chem. Soc. 129, 5515-5527 (2007).

19. Blasi, D., Nikolaidou, D. M., Terenziani, F., Ratera, I. \& Veciana, J. Excimers from stable and persistent supramolecular radical-pairs in red/NIR-emitting organic nanoparticles and polymeric films. Phys. Chem. Chem. Phys. 19, 9313-9319 (2017).

20. Peng, Q., Obolda, A., Zhang, M. \& Li, F. Organic light-emitting diodes using a neutral $\pi$ radical as emitter: the emission from a doublet. Angew. Chem. Int. Ed. 54, 7091-7095 (2015).

21. Ai, X. et al. Efficient radical-based light-emitting diodes with doublet emission. Nature 563, 536 (2018).

22. Neier, E. et al. Solution-processed organic light-emitting diodes with emission from a doublet exciton; using (2,4,6trichlorophenyl)methyl as emitter. Org. Electron. 44, 126-131, (2017).

23. Hattori, Y., Kusamoto, T. \& Nishihara, H. Luminescence, stability, and proton response of an open-shell (3, 5-dichloro-4pyridyl) bis (2, 4, 6-trichlorophenyl) methyl radical. Angew. Chem. Int. Ed. 53, 11845-11848 (2014).

24. Hattori, Y., Kusamoto, T. \& Nishihara, H. Enhanced luminescent properties of an open-shell (3,5-dichloro-4pyridyl)bis(2,4,6-trichlorophenyl)methyl radical by coordination to gold. Angew. Chem. Int. Ed. 54, 3731-3734 (2015). 
25. Kimura, S. et al. A luminescent organic radical with two pyridyl groups: high photostability and dual stimuli-responsive properties, with theoretical analyses of photophysical processes. Chem. Sci. 9, 1996-2007 (2018).

26. Guo, H. et al. High stability and luminescence efficiency in donor-acceptor neutral radicals not following the Aufbau principle. Nat. Mater. 18, 977-984 (2019).

27. Diez-Cabanes, V. et al. Design of perchlorotriphenylmethyl (PTM) radical-based compounds for optoelectronic applications: the role of orbital delocalization. ChemPhysChem. 19, 2572-2578 (2018).

28. He, C., Li, Z., Lei, Y., Zou, W. \& Suo, B. Unraveling the emission mechanism of radical-based organic light-emitting diodes. J. Phys. Chem. Lett. 10, 574-580 (2019).

29. Dewar, M. \& Longuet-Higgins, H. The electronic spectra of aromatic molecules I: Benzenoid hydrocarbons. Proc. Phys. Soc. A 67, 795 (1954).

30. Longuet-Higgins, H. \& Pople, J. A. The electronic spectra of aromatic molecules IV: Excited states of odd alternant hydrocarbon radicals and ions. Proc. Phys. Soc. A 68, 591-600 (1955).

31. Pople, J. A. Electron interaction in unsaturated hydrocarbons. Trans. Faraday Soc. 49, 1375-1385 (1953).

32. Pople, J. A. The electronic spectra of aromatic molecules II: A theoretical treatment of excited states of alternant hydrocarbon molecules based on self-consistent molecular orbitals. P. Phys. Soc. Lond. Section A. 68, 81 (1955).

33. Pariser, R. \& Parr, R. G. A. A semi-empirical theory of the electronic spectra and electronic structure of complex unsaturated molecules. I. J. Chem. Phys. 21, 466-471 (1953).

34. Pariser, R. Theory of the electronic spectra and structure of the polyacenes and of alternant hydrocarbons. J. Chem. Phys. 24, 250-268 (1956).

35. Dong, S. et al. Effects of substituents on luminescent efficiency of stable triaryl methyl radicals. Phys. Chem. Chem. Phys. 20, 18657-18662 (2018).

36. Franco, C. et al. Operative mechanism of hole-assisted negative charge motion in ground states of radical-anion molecular wires. J. Am. Chem. Soc. 139, 686-692 (2017).

37. Hele, T. J. H. et al. Anticipating acene-based chromophore spectra with molecular orbital arguments. J. Phys. Chem. A 123, 2527-2536 (2019)

38. Robinson, G. W. Intensity enhancement of forbidden electronic transitions by weak intermolecular interactions. J. Chem. Phys. 46, 572-585 (1967).

39. Li, W. et al. A hybridized local and charge-transfer excited state for highly efficient fluorescent OLEDs: Molecular design, spectral character and full exciton utilization. Adv. Opt. Mater. 2, 892-901 (2014).

40. Armet, O. et al. Inert carbon free radicals. 8. Polychlorotriphenylmethyl radicals: synthesis, structure, and spin-density distribution. J. Phys. Chem. 91, 5608-5616 (1987).

41. Fox, M. A., Gaillard, E. \& Chen, C. C. Photochemistry of stable free radicals: the photolysis of perchlorotriphenylmethyl radicals. J. Am. Chem. Soc. 109, 7088-7094 (1987).

42. Dong, S. et al. Multicarbazolyl substituted TTM radicals: red-shift of fluorescence emission with enhanced luminescence efficiency. Mater. Chem. Front. 1, 2132-2135 (2017).

43. Murawski, C. et al. Efficiency roll-off in organic light-emitting diodes. Adv. Mater. 25(47), 6801-6827 (2013).

44. Giebink, N. C. \& Forrest, S. R. Quantum efficiency roll-off at high brightness in fluorescent and phosphorescent organic light emitting diodes. Phys. Rev. B. 77(23), 235215 (2018).

45. Neese, F. The ORCA program system. Wiley Interdisciplinary Reviews - Computational Molecular Science. 2(1), 73-78 (2012). 


\section{Methods}

\section{Synthesis of the TTM-xPyID radicals}

The first stage of the TTM-xPyID synthesis required the preparation of TTM according to the previous report ${ }^{15}$. Next the pyridoindole groups $(9 H$-pyrido[2,3-b]indole $=\alpha$ PyID, $9 H$-pyrido[3,4- $b]$ indole $=\beta P y I D, 5 H-$ pyrido[4,3- $b]$ indole $=\gamma$ PyID and $5 H$-pyrido[3,2- $b]$ indole $=\delta$ PyID) were added to TTM in a mixture of DMF with $\mathrm{Cs}_{2} \mathrm{O}_{3}$, yielding a mixture of TTM-xPyID radicals and hydrogenated precursors, HTTM- $x$ PyID. The precursors were then deprotonated by KOtBu to obtain the carbanion variants, which were finally oxidised by $p$ chloroanil to obtain the target radicals: TTM- $\alpha$ PyID, TTM- $\beta$ PyID, TTM- $\gamma$ PyID and TTM- $\delta$ PyID. Full details of the synthesis can be found in SI 1.

\section{Photophysics}

Ultraviolet-visible (UV-vis) absorption spectra were recorded using a Shimadzu UV-2550 spectrophotometer. Photoluminescence (PL) spectra of the radicals were obtained with a Shimadzu 5301PC spectrofluorophotometer. An Edinburgh Instruments fluorescence spectrometer (FLS980) was used to measure the PL lifetimes and quantum yields of radicals in solution.

\section{Electronic structure calculations}

DFT calculations were performed with the ORCA package ${ }^{45}$ (version 4.0.1.2) using the B3LYP functional and 6-31G** basis set. For the open-shell radicals, unrestricted Kohn Sham (UKS) method was employed and the geometries were optimised in vacuo. The $\mathrm{D}_{1}$ excited state was evaluated by UKS-time-dependent-DFT with the Tamm-Dancoff approximation.

\section{Device fabrication and characterisation}

The radical organic light-emitting diodes (OLEDs) were fabricated by vacuum deposition processing (pressure $<6 \times 10^{-7}$ Torr) using an Angstrom Engineering EvoVac 700 system.

Current density-voltage-electroluminescence (J-V-EL) characteristics were measured using a Keithley 2400 sourcemeter, Keithley 2000 multimeter and calibrated silicon photodiode. The EL spectra were recorded by an Ocean Optics Flame spectrometer. The transient PL and EL studies on radical-based films and OLEDs were 
conducted on a time-correlated single photon counting (TCSPC) setup: Edinburgh Instruments Life Spec system with Hammamatsu photomultiplier tube. A function generator (8116A Hewlett Packard) was used for electrical excitation; a picosecond $407 \mathrm{~nm}$ laser (PicoQuant 407, pulse width $=50 \mathrm{ps}$ ) was used for optical excitation. The electrical and optical excitation pulses for OLED measurements were synchronised by a digital delay generator (Stanford Research Systems, DG 645), which also triggered the TCSPC setup. 